GRADIATION\&APPLICATIONS

ISSN 2466-4294 (online) | rad-journal.org

Vol. 3 | Issue 3 | pp. 165-171, 2018

doi: 10.21175/RadJ.2018.03.028

Original research paper

\title{
TEMPERATURE STABILIZATION OF SIPM-BASED GAMMA-RADIATION SCINTILLATION DETECTORS*
}

\author{
Viktors Ivanovs**, S. Gushchin, Valerijs Ivanovs, \\ V. Fjodorovs, D. Kuznecovs, A. Loutchanski, V. Ogorodniks
}

\author{
ZRF RITEC SIA, Riga, Latvia
}

\begin{abstract}
Silicon photomultipliers (SiPMs) coupled with various scintillators are currently used as gammaradiation detectors for different applications. Many tasks require the ability to use detectors in environments with varying operating temperatures. However, the profound dependences of the characteristics of both SiPMs and scintillators on temperature make it difficult to use these detectors in such environmental conditions. The gain of an SiPM increases with increases in bias voltage, and it decreases with increases in temperature; however, the scintillator's light yield may increase and/or decrease with temperature, depending on the type of scintillator used. Such temperature dependence makes it necessary to use special techniques for the stabilization of the detector parameters. We proposed and tested a method and an electronic module for compensating for the temperature instabilities of the gain of an SiPM and the light output of BGO and CsI(Tl) scintillators. Our method is based on the application of the SiPM biasing power supply that is controlled and managed by the microprocessor. The calibration data of the temperature dependence of a photo peak $(662 \mathrm{keV})$ are stored in the microprocessor memory. The exact value of the bias voltage for each temperature is calculated by the formula of the 5th-degree polynomial. This method achieved a high accuracy of the photo peak position stabilization in the tested operation temperature range $\left(-2 O^{\circ} \mathrm{C}-\right.$ $+50^{\circ} \mathrm{C}$ ). The test results of the SiPM-based gamma-radiation BGO and CsI(Tl) scintillation detectors as well as the results of their practical applications in medical surgical probes are presented.
\end{abstract}

Key words: Scintillation detector, BGO, CsI(Tl), gamma-radiation detector, SiPM, silicon photomultiplier, temperature stabilization

\section{INTRODUCTION}

Silicon photomultipliers (SiPMs) are good alternatives to traditional photomultiplier tubes for application in the scintillation detectors used to convert optical signals to electrical ones and to amplify them to measured values $[1,2]$.

SiPMs are a set of miniature avalanche diodes operating in Geiger mode and are connected in parallel. The diode and the quenching resistor comprise a micro cell of the SiPM. SiPMs have a smaller size and a similar gain compared to those of traditional vacuum tube-based photomultipliers.

SiPMs are of a more compact size, they have a higher reliability and stability under mechanical impacts, and a lower sensitivity to magnetic fields, and they can operate at significantly lower operating voltages compared to traditional photomultipliers. The use of SiPMs with scintillation detectors makes it possible to obtain a satisfactory energy resolution for many applications.
However, SiPMs have some disadvantages. In addition to an increased noise level, the characteristics of SiPMs, primarily the gain, significantly depend on temperature. Changes in the characteristics of SiPMs caused by temperature changes limit their application in conditions with a changing operating temperature of the environment, for example, various field conditions, homeland security conditions, and military conditions.

In addition to the temperature instability of SiPMs, the dependence of the characteristics of scintillators on temperature can also limit the use of SiPM-based scintillation detectors.

The influence of the instability of the electronics used with an SiPM on temperature also has a negative effect on the stability of the detector characteristics.

This paper considers the influence of temperature on the characteristics of detectors based on inorganic CsI(Tl) and BGO scintillators produced by the company EPIC Crystals Co., LTD. [3] as well as a SiPM produced by the company SensL [4], and it suggests a method and a device for compensating for the temperature dependences.

\footnotetext{
* This paper was presented at the Sixth International Conference on Radiation and Applications in Various Fields of Research (RAD 2018), Ohrid, Macedonia, 2018.

*nitec@ritec.lv
} 
2. DEPENDENCE OF CHARACTERISTICS OF SiPMS AND SCINTILLATORS ON TEMPERATURE AND METHODS OF THEIR COMPENSATION

\subsection{Dependence of $\mathrm{CsI}(\mathrm{Tl})$ and $B G O$ scintillators on temperature}

It is known that scintillator characteristics, such as the light yield (or scintillation efficiency) and the light pulses that decay over time, depend on temperature. In addition, the luminescence spectrum also depends on temperature; however, the amount of change is insignificant and has a slight effect on work stability. The light yield in BGO scintillators decreases substantially with decreasing temperature $[5,6,7,8]$. In the temperature range from $-20^{\circ} \mathrm{C}$ to $+50^{\circ} \mathrm{C}$, the light yield in BGO scintillators depends almost linearly on temperature. According to data from various publications the temperature coefficient of the light yield at temperatures close to room temperature is $-0.9 \% /{ }^{\circ} \mathrm{C}[8]$ or $-1.2 \% /{ }^{\circ} \mathrm{C}[5]$. The light pulse decay time in BGO scintillators varies from approximately $0.6 \mu \mathrm{s}$ at $-20^{\circ} \mathrm{C}$ to approximately $0.2 \mu \mathrm{s}$ at $+50^{\circ} \mathrm{C}[7]$. The maximum emission spectrum in BGO crystals corresponds to $480 \mathrm{~nm}$.

The light yield in CsI(Tl) scintillators depends on the temperature to a lesser degree than in BGO scintillators; however, the behavior is different. There is a maximum light yield lying in the temperature range from $-40^{\circ} \mathrm{C}$ to $+30^{\circ} \mathrm{C}[9,10]$. The temperature coefficient of the light yield at temperatures close to room temperature is approximately $0.4 \% /{ }^{\circ} \mathrm{C}[8]$. The light pulse decay time in CsI(Tl) scintillators also depends on temperature. However, the decay time in these crystals consists of more than one component. The fast and slow components at room temperature have a decay time of approximately $0.6 \mu \mathrm{s}$ and $3.5 \mu \mathrm{s}$, respectively [10]. The maximum of the emission spectrum in CsI(Tl) crystals corresponds to $550 \mathrm{~nm}$.

BGO detectors have a higher gamma-ray absorption efficiency and a shorter light pulse decay time but a much lower light yield and a lower energy resolution compared to $\mathrm{CsI}(\mathrm{Tl})$ detectors. BGO detectors are recommended for use in tasks where high efficiency of radiation detection is required whereas high energy resolution is not.

The dependence of the light pulse decay time on temperature determines the change in the shape of the output signals of the detector with temperature changing. This phenomenon leads to an additional change in the position of the centroids of the detected photo peaks due to the ballistic deficit in the shaping amplifier caused by a change in the relation between the light pulse decay time of scintillators and the amplifier shaping time [9].

\subsection{Dependence of the SiPM characteristics on operating temperature}

SiPMs are solid-state devices based on a silicon semiconductor material. The characteristics of such devices profoundly depend on temperature. It is the leakage current that determines the level of noise, breakdown voltage, gain, junction capacitance, optical crosstalk, after-pulsing and photon registration efficiency $[11,12,13,14]$. However, the most important parameter of the SiPM that depends on temperature is the gain. The essential dependence of the gain on temperature is related to the dependence of the breakdown voltage on temperature. The dependence of the breakdown voltage on temperature is $21.5 \mathrm{mV} /{ }^{\circ} \mathrm{C}$, and the gain of SiPM C-series produced by SensL is $0.8 \% /{ }^{\circ} \mathrm{C}[11,15]$.

Moreover, the gain of SiPMs depends on the overvoltage. With an increase in the overvoltage, the gain of the SiPM also increases. It is possible to compensate the gain temperature dependence using this behavior.

2.3. Methods for the compensation of temperature dependences of the characteristics of detectors

based on scintillators and SiPMs

Both the temperature shift of an SiPM gain and electronics and the change in the light yield in the scintillator substantially deteriorate the spectrometric characteristics of the measuring equipment when the measurements are conducted in a relatively large time interval under conditions of varying operating temperature. To eliminate this deterioration, it is necessary, in one way or another, to stabilize the detector characteristics.

In the literature, several approaches for stabilizing the SiPM gain under conditions of changing environmental temperature have been described. Some of these approaches were previously used to stabilize the characteristics of conventional vacuum-tube-based photomultipliers coupled with scintillator detectors $[16,17]$. These are methods based on the use of various electronics, radioactive sources incorporated into the material of a scintillator [18], or pulsed light-emitting diodes $[19,20]$.

There are also other methods when specific features of SiPMs [21 - 26] are used.

The most obvious approach is to stabilize the characteristics of the detector by stabilizing the operating temperature, for example, cooling the detector, using the Peltier element [27] or using other methods of the temperature stabilization of the detector [28]. However, these methods require complex design solutions for their implementation that are energy-consumable and that cannot be used in portable devices for field application. Other methods $[21,22,23]$ are based on the use of the temperature dependence of the SiPM gain and the temperaturedependent bias voltage power supply. These methods mainly differ in the methods of operating temperature measurement.

Some of the proposed methods allow the compensation of only the temperature dependence of the SiPM gain but not the temperature dependence of the scintillator characteristics and the temperature instability of the electronics.

In reference [23], the temperature stabilization of the SiPM gain was conducted using the method of active monitoring of a bias voltage. The developed experimental setup was controlled via PC.

We also proposed a method and a device for temperature dependence compensation of SiPM-based 
scintillation detector characteristics based on a controlled bias power supply.

\section{EXPERIMENT}

\subsection{Detectors}

To demonstrate the developed scheme of temperature compensation, we selected BGO and CsI(Tl) scintillation detectors that have different temperature dependences of their characteristics. Scintillators of different sizes were used.

To build the detector modules, SensL low noise Cseries SiPM [15] MicroFC 60035, with a sensitive area of $6 \times 6 \mathrm{~mm}^{2}$ and a microcell size of $35 \mu \mathrm{m}$, as well as a MicroFC30035 with a sensitive area of $3 \times 3 \mathrm{~mm}^{2}$ and a microcell size of $35 \mu \mathrm{m}$, were used. The breakdown voltage of these SiPMs at room temperature is approximately $24.5 \mathrm{~V}$, and the recommended overvoltage range is $1.0 \mathrm{~V}$ to $5.0 \mathrm{~V}$.

The detector module consisted of three components: a scintillator (BGO or CsI(Tl)), an SiPM, and a thermistor. The SiPM and the thermistor were mounted on a common PCB. The close arrangement of the thermistor and the SiPM provided an accurate measurement of the SiPM temperature and, accordingly, an accurate setting of the operating bias voltage to compensate for temperature changes in its characteristics.

The scintillator and SiPM were coupled together by a low-stress optical epoxy resin EPO-TEK 301-2FL. Further, a diffusion reflector covered the resulting assembly. A Teflon film was used as a diffusion reflector. tests:

The following detectors were assembled for the

1. Scintillator BGO $\varnothing_{12.5} \mathrm{~mm} \times 50 \mathrm{~mm}, \mathrm{SiPM}$ MicroFC 60035.

2. Scintillator CsI (Tl) Ø12.0 $\mathrm{mm} \times 50 \mathrm{~mm}, \mathrm{SiPM}$ MicroFC 60035.

3. Scintillator BGO $\varnothing 6 \mathrm{~mm} \times 10 \mathrm{~mm}, \mathrm{SiPM}$ MicroFC 30035.

4. Scintillator CsI (Tl) $\varnothing 6 \mathrm{~mm} \times 10 \mathrm{~mm}, \mathrm{SiPM}$ MicroFC 30035.

The appearance of the assembled detector modules is shown in Fig. 1.

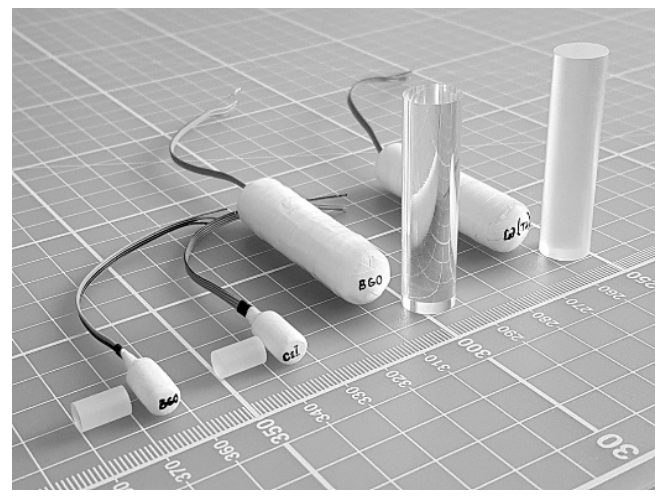

Figure 1. The assembled detector modules
3.2. Method of compensation of the temperature dependences

The temperature stabilization of the parameters of the assembled detectors was conducted using the method of active monitoring of the detector bias voltage. This method requires precise preliminary calibration. Calibration allows compensating not only for the effect of temperature instability of the SiPM gain and for the light yield of the scintillator but also for the temperature instability of the scintillator light pulse decay time. In addition, calibration allows taking into account the temperature instability of electronic circuits (amplifier and bias power supply) if they are arranged in close proximity to the detector.

The temperature calibration of the detectors was conducted with the use of a temperature chamber, which allowed for setting and maintaining the temperature, with an accuracy of $\pm 0.25^{\circ} \mathrm{C}$.

For the measurements, the detector was connected to the measuring equipment - digital multichannel analyzer MCA527.

Based on the requirements for parameters such as optimal signal-to-noise ratio, dynamic range of the control system in the operating temperature range $-20^{\circ} \mathrm{C}-+50^{\circ} \mathrm{C}$, the reference voltage of $\mathrm{SiPM} 28,5 \mathrm{~V}$ for the temperature point of $25^{\circ} \mathrm{C}$ was selected.

To improve the calibration accuracy, the entire process must be performed in one continuous measurement cycle for each detector. To ensure the operating temperature range of the system from $-20^{\circ} \mathrm{C}$ to $+50^{\circ} \mathrm{C}$, the calibration in an extended temperature range $-25^{\circ} \mathrm{C}-+55^{\circ} \mathrm{C}$ was performed. Based on the capabilities of the equipment used, within 1 working day it was possible to carry out measurements in 9 temperature points of the extended temperature range in step of $10^{\circ} \mathrm{C}$.

The following calibration procedure was used.

First of all, for each of the selected temperature points, the bias voltage of the SiPM was manually adjusted so that the peak of $662 \mathrm{keV}$ was in the region of the target channel with an accuracy of $\pm 0.1 \%$. The target channel is a channel of maximum of ${ }^{137 \mathrm{Cs}} 662$ $\mathrm{keV}$ photo peak recorded at $25^{\circ} \mathrm{C}$ and at bias voltage of $28.5 \mathrm{~V}$. The current operating temperature was controlled by a temperature sensor - the NTC thermistor arranged near the SiPM.

The experimental dependence of the adjusted bias voltage as a function of temperature was obtained.

Then, this dependence was approximated by a $5^{\text {th }}$ order polynomial dependence (calibration curve). The resulting polynomial can be used by a microcontroller of the stabilization system to calculate the required operating voltage at any measured temperature point, however, to reduce the calculation time, the following procedure was implemented.

Dependences of the calculated bias voltage for different detectors on temperature are shown in Fig. 2.

According to the equations of the polynomials, the values of the bias voltage of SiPM for the temperature range from $-25^{\circ} \mathrm{C}$ to $+55^{\circ} \mathrm{C}$ in steps of $5^{\circ} \mathrm{C}$ were calculated and tabulated into the memory of the microprocessor, which controls the temperature 
stabilization unit. The bias voltage for all intermediate temperature values are calculated by the microcontroller of the stabilization system using linear interpolation. An identical calibration procedure has been applied to all four types of detectors.

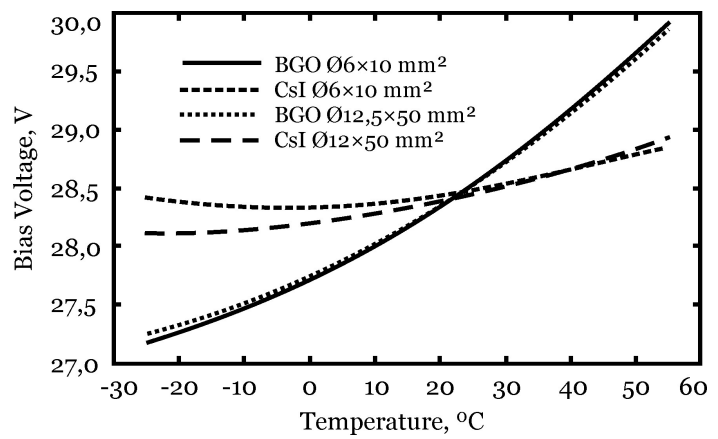

Figure 2. Dependence of the calculated bias voltage for different detectors on temperature used for gain stabilization

\subsection{The proposed scheme}

The architecture of the temperature stabilization scheme of the detector is shown in Fig. 3.

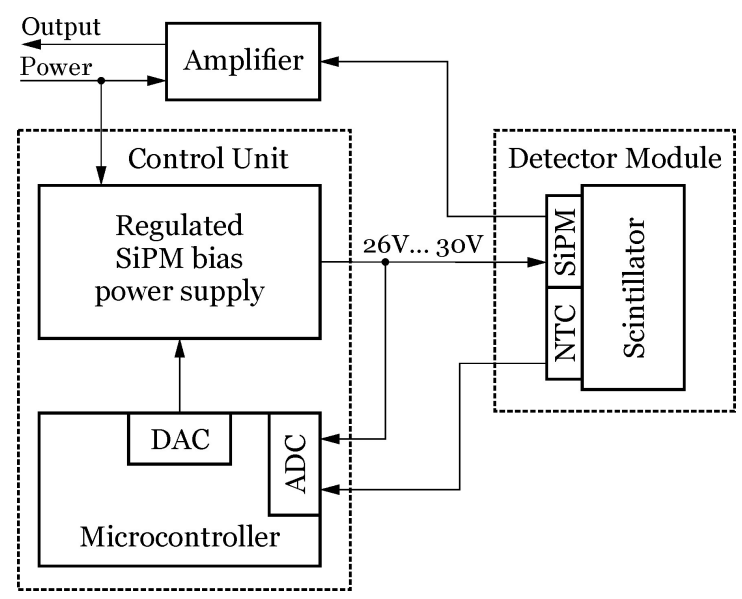

Figure 3. Block diagram of the detector's temperature compensation scheme

The microcontroller MKLo5Z32VFK4, based on the Cortex-Mo+ core, which includes 12-bit ADCs and DACs, achieves the operation control of the entire circuit. The microcontroller periodically measures the current temperature of the detector.

Based on the calibration table stored in the processor memory, the required bias voltage of the detector is determined. There is a voltage set at the DAC output of the microcontroller that controls the regulated bias power supply of the SiPM. The bias power supply is based on the LT3571EUD chip. The circuit is designed in such a manner that, when the voltage of the DAC output is changed from o V to $2.5 \mathrm{~V}$, the voltage of the output of the detector bias source varies from $26 \mathrm{~V}$ to $30 \mathrm{~V}$. The accuracy of setting the required bias voltage of the detector is $\pm 4 \mathrm{mV}$, and the accuracy of maintaining the set value is $\pm 1 \mathrm{mV}$. This accuracy of setting the bias voltage makes it possible to maintain the accuracy of keeping the amplitude of the output signal of the detector within $\pm 0.5 \%$.

The microcontroller also periodically controls the correctness setting of the bias voltage of the detector.

The SiPM is connected to the amplifier by the standard mode positive biasing and AC coupled readout [15], but without fast output. Such connection allows excluding the shift of the baseline at the output of the amplifier caused by the temperature change in the dark current of the SiPM.

The amplifier based on the ADA4805-1ARJZ chip amplifies and shapes the signals. A pseudo-Gaussian waveform at the output of the amplifier has a duration at the half-height of the signal, approximately $1.1 \mu \mathrm{s}$. The signal from the detector through the amplification stage is sent to the subsequent recording equipment.

All the elements of this scheme are located on two small PCBs with dimensions of $45 \mathrm{~mm} \times 12 \mathrm{~mm}$ and $39 \mathrm{~mm} \times 7 \mathrm{~mm}$, which are coupled to each other by a connector. The small size of the developed module allows their arrangement in a cylindrical case with an internal diameter of $14 \mathrm{~mm}$.

The power supply voltage of the module is $+5 \mathrm{~V}$, and the current consumption does not exceed $12 \mathrm{~mA}$ in the entire tested temperature range.

The appearance of the PCBs is shown in Fig. 4.

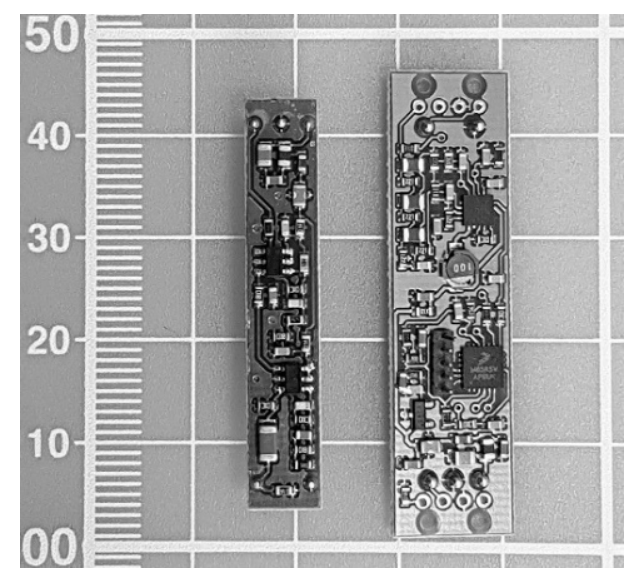

Figure 4. PCBs of the temperature compensation module

\section{TEST RESUltS}

The developed scheme for the compensation of the temperature dependence of the characteristics of detectors is based on the scintillator and SiPM tests in the temperature range from $-20^{\circ} \mathrm{C}$ to $+50^{\circ} \mathrm{C}$ using the temperature chamber. The tests were conducted in both a stationary mode (when the measurements were started after maintaining a given temperature for $\mathbf{1}$ hour) and in a dynamic mode, with a continuous change in temperature in the measuring chamber at the speed of approximately $1.8^{\circ} \mathrm{C} / \mathrm{min}$.

Figure 5 and Figure 6 show the position of the photo peak $662 \mathrm{keV}$ centroid and the energy resolution (FWHM) in the temperature range with and without the use of the stabilization scheme, as measured with $\mathrm{BGO}$ and $\mathrm{CsI}(\mathrm{Tl})$ detectors of different sizes. 
V. Ivanov et al., Temperature stabilization of SiPM-based..., Rad. Applic., 2018, 3, 3, 165-171

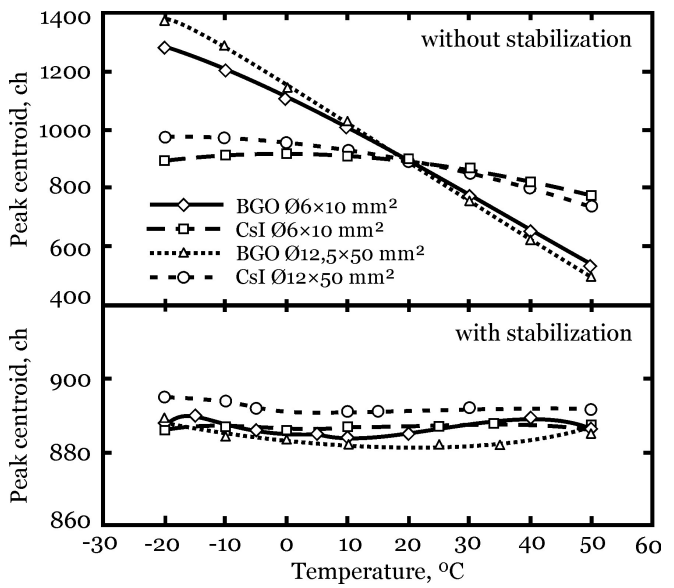

Figure 5. Dependence of the photo peak at $662 \mathrm{keV}$ centroid on temperature with and without using the stabilization scheme measured with different detectors

The change in the position of the photo peak centroid in the tested temperature range for BGO detectors without the use of stabilization was significant. The amplitude of the signals decreased approximately by a factor of 2 in this temperature range. The change in the position of the photo peak centroid in the same temperature range for the $\mathrm{CsI}(\mathrm{Tl})$ detectors without using the stabilization system was not so significant as for the BGO detectors. The amplitude of the signal decreased in this temperature range approximately 1.2 -fold compared with the amplitude at room temperature.

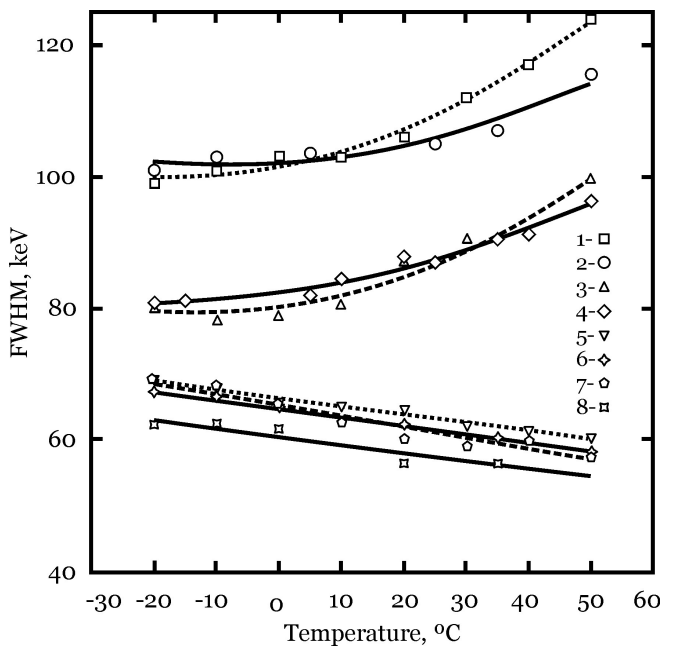

Figure 6. Dependence of the energy resolution at $662 \mathrm{keV}$ on temperature without $(1,3,5,7)$ and with using $(2,4,6,7)$ the stabilization scheme measured with the different detectors: 1, 2 - BGO Ø12.5 mm × 50 mm, 3, 4 - BGO Ø6 mm $\times 10 \mathrm{~mm}$, $5,6-\mathrm{CsI}(\mathrm{Tl}) \varnothing 12.0 \mathrm{~mm} \times 50 \mathrm{~mm}$ 7,8 - CsI (Tl) Ø6 mm × $10 \mathrm{~mm}$.

The use of the temperature compensation circuit allowed us to stabilize the peak positions for all types of detectors.

Changes in the photo peak position did not exceed $0.07 \% /{ }^{\circ} \mathrm{C}$. The measurements in a dynamic mode with constantly changing operating temperature (approximately $1.8^{\circ} \mathrm{C} / \mathrm{min}$ ) yielded the same results.

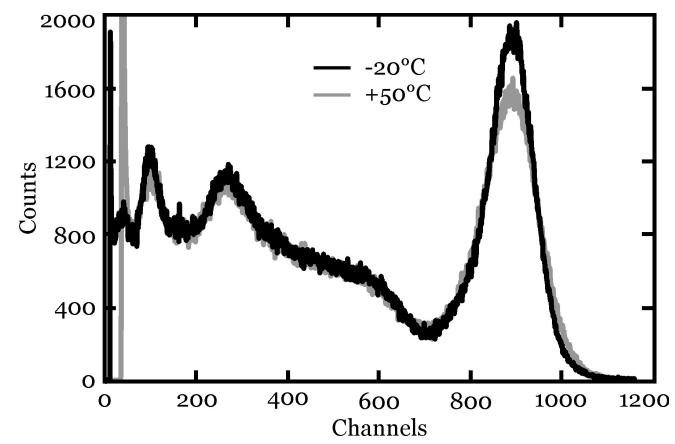

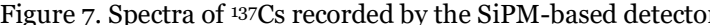
with a BGO scintillator with the size of $\varnothing 6 \mathrm{~mm} \times 10 \mathrm{~mm}$ at different operation temperatures

At the same time, the use of the compensating scheme did not significantly affect the dependences of energy resolution on temperature. Both with and without the application of the compensating scheme, the energy resolution of the detectors with the CsI(Tl) scintillators deteriorated with decreasing temperature, and that of the detectors with the BGO scintillators improved with decreasing temperature. Such dependences are primarily caused by the difference in the behavior of the light yield and light pulse decay time in these detectors on temperature.

The ${ }^{137} \mathrm{Cs}$ spectra obtained by detectors with the BGO scintillators with the size of $\varnothing 6 \mathrm{~mm} \times 10 \mathrm{~mm}$ at different temperatures are shown in Fig. 7.

The parameters of the developed correction scheme and the results of its testing demonstrate the possibility of their application in devices intended for the use in conditions of changing ambient temperature.

For example, the developed correction scheme was used to stabilize the characteristics of a surgical probe intended for the measurement of gamma-radiation from isotopes injected into the human body for cancer diagnostics. The appearance of the probe is shown in Fig. 8.

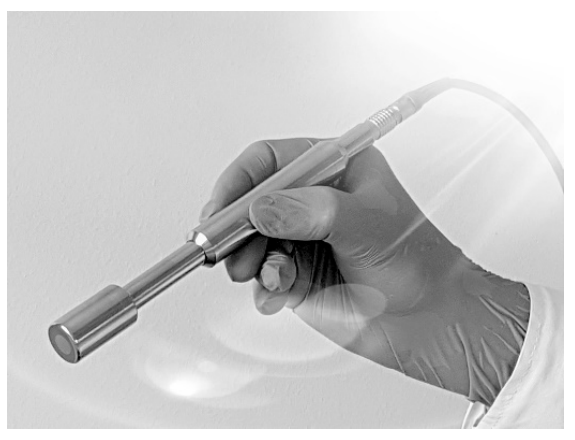

Figure 8. The surgical gamma-radiation probe

The surgical probe consists of an SiPM-based BGO scintillator detector with the size of $\varnothing 6 \mathrm{~mm} \times 10 \mathrm{~mm}$, a tungsten collimator, a thermal compensation scheme and a shaping amplifier. The external dimensions of the probe are as follows: head diameter $-17 \mathrm{~mm}$, head 
V. Ivanov et al., Temperature stabilization of SiPM-based..., Rad. Applic., 2018, 3, 3, 165-171

length $-30 \mathrm{~mm}$, and total probe length $-165 \mathrm{~mm}$. The probe is designed for application in the temperature range from $+10^{\circ} \mathrm{C}$ to $+40^{\circ} \mathrm{C}$. The gain stability for the temperature range is $<0.6 \%$.

After careful calibration, the temperature stabilization of the detectors shows good repeatability. A total of 50 similar-type detectors using the same calibration curve were fabricated and tested. The scattering in the photo peak of $662 \mathrm{keV}$ centroid position in the temperature range from $+10^{\circ} \mathrm{C}$ to $+40^{\circ} \mathrm{C}$ for this batch was $\pm 1.8 \%$.

\section{CONCLUSION}

A temperature compensation module using the method of active monitoring of the bias voltage of a detector was developed and tested with SiPM-based $\mathrm{BGO}$ and $\mathrm{CsI}(\mathrm{Tl})$ scintillator detectors of different sizes.

The method of accurate calibration of the stabilization unit allows for compensating the temperature instabilities of scintillation detectors, SiPMs and electronic components.

The developed firmware for the microprocessor provides control and management of the fabricated module.

The proposed method and the developed temperature stabilization module demonstrate a significant improvement in the temperature stability of the tested detectors. The temperature coefficient of the photo peak of $662 \mathrm{keV}$ in the temperature range from $20^{\circ} \mathrm{C}$ to $+50^{\circ} \mathrm{C}$ is approximately $0.07 \% /{ }^{\circ} \mathrm{C}$ for all types of the tested detectors.

The developed temperature-compensating module was used in a small-scale production of surgical gamma-radiation probes. The experience of using the developed modules in the production of these probes has shown the possibility of using the same calibration table for the same type of detectors, with high reproducibility of the results.

\section{REFERENCES}

1. B. Sanaei, M. T. Baei, Z. Sayyed-Alangi, "Characterization of a New Silicon Photomultiplier in Comparison with a Conventional Photomultiplier Tube," J. Modern Phys., no. 6, pp. 425 - 433, Mar. 2015.

Retrieved from: https://file.scirp.org/pdf/JMP 2015032514312660.pdf Retrieved on: Nov. 23, 2018

2. M. A. Wonders, D. L. Chichester, M. Flaska, "Characterization of New-Generation Silicon Photomultipliers for Nuclear Security Applications," in EPJ Web Conf., Advancements in Nuclear Instrumentation Measurement Methods and their Applications (ANIMMA 2017), Liège, Belgium, Jun. 2017.

Retrieved from: https://www.epjconferences.org/articles/epjconf/pdf/2018/05/epjconf animma2018 07015.pdf;

Retrieved on: Nov. 23, 2018

3. Download Center, EPIC Crystals Co., Ltd., Kunshan, China, 2018

Retrieved from: http://www.epic-

crystal.com/download-center/;
Retrieved on: Nov. 23, 2018

4. Product Overview, C-SERIES SIPM: Silicon Photomultiplier Sensors, SensL, Cork, Ireland, 2018. Retrieved from: https://www.onsemi.com/ PowerSolutions/product.do?id=CSERIES\%20SIPM\&pdf=Y; Retrieved on: Nov. 23, 2018

5. BGO Bismuth Germanate Scintillation Material, Data Sheet, Saint-Gobain Crystals, La Défense, France, 2018. Retrieved from: https://www.crystals.saintgobain.com/sites/imdf.crystals.com/files/documents/b go-material-data-sheet.pdf; Retrieved on: Nov. 23, 2018

6. P. L. Wang, Y. L. Zhang, Z. Z. Xu, X. L. Wang, "Study on the temperature dependence of BGO light yield," Sci. China Phys. Mech., vol. 57, no. 10, pp. 1898 - 1901, Jun. 2014.

DOI: $10.1007 / \mathrm{s} 11433-014-5548-4$

7. C. L. Melcher, J. S. Schweitzer, A. Liberman, J. Simonetti, "Temperature dependence of fluorescent decay time and emission spectrum of bismuth germinate," IEEE Trans. Nucl. Sci., vol. NS-32, no. 1, pp. $529-532$, Feb. 1985. DOI: $10.1109 /$ TNS.1985.4336887

8. R. Mao, L. Zhang, R.-Y. Zhu, "Optical and Scintillation properties of inorganic scintillators in high energy physics, IEEE Trans. Nucl. Sci., vol. 55, no. 4, pp. 2425 - 2431, Aug. 2008. DOI: 10.1109/TNS.2008.2000776

9. J. D. Valentine, W. W. Moses, S. E. Derenzo, D. K. Wehe, G. F. Knoll, "Temperature dependence of CsI(Tl) gamma-ray excited scintillation characteristics," Nucl. Instr. Meth. Phys. Res., vol. 325, pp. 147 - 157, Feb. 1993.

DOI: 10.1016/0168-9002(93)91015-F

10. CsI(Tl), CsI(Na) Cesium Iodide scintillation material, Data Sheet, Saint-Gobain Crystals, La Défense, France, 2018.

Retrieved from: https://www.crystals.saint-

gobain.com/sites/imdf.crystals.com/files/documents/c sitl-and-na-material-data-sheet.pdf;

Retrieved on: Nov. 23, 2018

11. Introduction to SiPM, Technical note, Rev. 6.o, SensL, Cork, Ireland, 2017.

Retrieved from: https://www.sensl.com/downloads/ds/ TN\%20-\%20Intro\%20to\%20SPM\%20Tech.pdf; Retrieved on: Nov. 23, 2018

12. S. Piatek, How does temperature affect the gain of an SiPM? Hamamatsu Corporation \& New Jersey Institute of Technology, New Jersey (NJ), USA, 2016.

Retrieved from: https://hub.hamamatsu.com/sp/hc/re sources/Temperature Gain SiPM.pdf?utm source $=\mathrm{hc}$ \&utm $\_$medium $=$email\&utm campaign=hc-enews; Retrieved on: Nov. 23, 2018

13. P. Eckert, H.-C. Schultz-Coulon, W. Shen, R. Stamen, A. Tadday, "Characterisation studies of silicon photomultipliers," Nucl. Instrum. Methods Phys. Res. vol. 620, no. 2-3, pp. 217 - 226, Aug. 2010. DOI: $10.1016 / \mathrm{j}$.nima.2010.03.169

14. M. Ramilli, "Characterization of SiPM: temperature dependencies," in Proc. 2008 IEEE Nuclear Science Symposium (NSS/MIC), Dresden, Germany, 2008. DOI: 10.1109/NSSMIC.2008.4774854

15. Datasheet: Silicon Photomultipliers (SiPM), Low Noise, Blue-Sensitive, SensL, Cork, Ireland, 2018. Retrieved from: https://www.onsemi.com/pub/Collater al/MICROC-SERIES-D.PDF; Retrieved on: Nov. 23, 2018

16. A. Manor at al., "Compensation of scintillation sensor gain variation during temperature transient conditions using signal processing techniques," in Proc. 2009 IEEE Nuclear Science Symposium Conference Record 
V. Ivanov et al., Temperature stabilization of SiPM-based..., Rad. Applic., 2018, 3, 3, 165-171

(NSS/MIC), Orlando (FL), USA, 2009, pp. 2399 2403.

DOI: 10.1109/NSSMIC.2009.5402169

17. G. Pausch, J. Stein, N. Teofilov, "Stabilizing scintillation detector system by exploiting the temperature dependence of the light pulse decay time," IEEE Trans. Nucl. Sci., vol. 52, no. 5, pp. $1849-1855$, Oct. 2005 .

DOI: 10.1109/TNS.2005.856616

18. R. W. Carlson, "Standardized luminophore," U.S. patent US3030509, USA, Apr. 17, 1962.

Retrieved from: https://patentimages.storage.googleapi s.com/11/8b/98/65ada86acbf647/US3030509.pdf; Retrieved on: Nov. 23, 2018

19. K. Saucke, G. Pausch, J. Stein, H.-G. Ortlepp, P. Schotanus, "Stabilizing scintillation detector systems with pulsed LEDs: a method to derive the LED temperature from pulse height spectra," IEEE Trans. Nucl. Sci., vol. 52, no. 6, pp. 3160 - 3165, Dec. 2005. DOI: 10.1109/TNS.2005.862929

20. M. Yamashita, S. Takeuchi, "Temperaturecompensating pulsed reference light source using a LED," Rev. Sci. Instrum., vol. 54, no. 12, pp. 1795 1796, Aug. 1983. DOI: $10.1063 / 1.1137342$

21. A. V. Stolin, S. Majewski, R. R. Raylman, "Novel Method of Temperature Stabilization for SiPM-Based Detectors," IEEE Trans. Nucl. Sci., vol. 6o, no. 5, pp. $3181-3187$, Oct. 2013. DOI: 10.1109/TNS.2013.2273398

22. Z. Li et al., "A gain control and stabilization technique for silicon photomultipliers in low-light-level applications around room temperature", Nucl. Instr.
Meth. Phys. Res. A, vol. 695, spec. issue, pp. $222-225$, Dec. 2012.

DOI: 10.1016/j.nima.2011.12.037

23. F. Licciulli, C. Marzocca, "An Active Compensation System for the Temperature Dependence of SiPM Gain," IEEE Trans. Nucl. Sci., vol. 62, no. 1, pp. $228-235$, Feb. 2015. DOI: $10.1109 /$ TNS.2015.2388580

24. P. S. Marrocchesi et al., "Active control of the gain of a 3 mm x $3 \mathrm{~mm}$ silicon photomultiplier," Nucl. Instr. Meth. Phys. Res. Sec. A, vol. 602, no. 2, pp. 391 - 395, 2009. DOI: 10.1016/j.nima.2008.12.199

25. G. Eigen et al., "SiPM gain stabilization studies for adaptive power supply," presented at the International Workshop on Future Linear Colliders (LCWS15), Whistler, Canada, 2015.

Retrieved from: https://arxiv.org/pdf/1603.00016.pdf; Retrieved on: Nov. 23, 2018

26. A. Kaplan, "Correction of SiPM temperature dependencies," Nucl. Instr. Meth. Phys. Res. A, vol. 610, no. 1, pp. 114 - 117, Oct. 2009. DOI: 10.1016/j.nima.2009.05.137

27. S. Nieswand, "A Peltier cooling system for SiPM temperature stabilization,” B.Sc. dissertation, CERN Geneva, Switzerland, Oct. 2012. Retrieved from: https://web.physik.rwthaachen.de/ hebbeker/theses/nieswand bachelor.pdf; Retrieved on: Nov. 23, 2018

28. G. Collazuol, M. G. Bisognia, S. Marcatilia, C. Piemonte, A. Del Guerraa, "Studies of silicon photomultipliers at cryogenic temperatures," Nucl. Instr. Meth. Phys. Res. Sec. A, vol. 628, no. 1, pp. 389 - 392, Feb. 2011. DOI: $10.1016 /$ j.nima.2010.07.008 